FORSCHUNGSBERICHTE DES LANDES NORDRHEIN-WESTFALEN

Nr. 3226 / Fachgruppe Wirtschafts- und Sozialwissenschaften

Herausgegeben vom Minister für Wissenschaft und Forschung

Dip1.-Ökonomin Hannelore Neumann

Forschungsinstitut der Friedrich-Ebert-Stiftung

Bonn - Bad Godesberg

Frauen in der öffentlichen

Arbeitsmarkt- und Strukturpolitik

州

Westdeutscher Verlag 1988 
EDV: Frank Weimer

Textverarbeitung: Barbara Becker

CIP-Titelaufnahme der Deutschen Bibliothek

Neumann, Hannelore:

Frauen in der öffent 1 ichen Arbeitsmarktund Strukturpolitik / Hannelore Neumann. Opladen: Westdt. Ver1., 1988

(Forschungsberichte des Landes Nordrhein-

Westfalen; Nr. 3226 : Fachgruppe

Wirtschafts - und Sozialwissenschaften)

ISBN 978-3-531-03226-9 ISBN 978-3-322-87590-7 (eBook)

DOI $10.1007 / 978-3-322-87590-7$

NE: Nordrhein-Westfalen: Forschungsberichte des Landes

Der Westdeutsche Verlag ist ein Unternehmen der Verlagsgruppe Bertelsmann.

(c) 1988 by Westdeutscher Verlag GmbH, Opladen

Herstellung: Westdeutscher Verlag

ISBN 978-3-531-03226-9 


\author{
Verzeichnis der übersichten \\ Verzeichnis der Abbildungen \\ Verzeichnis der Tabellen
}

$X I$

1.

2.

Die Notwendigkeit arbeitsmarkt - und strukturpolitischer MaBnahmen für Frauen vor dem Hintergrund ihrer Situation in der Arbeitswelt

2.1 Offene und versteckte Arbeitslosigkeit von Frauen. Sind Frauen gar nicht richtig arbeitslos?

2.2 Erklärungsmuster für das überdurchschnittliche Arbeitslosigkeitsrisiko von Frauen

2.2.1 Wunsch nach Teilzeitarbeit oder Zwang zur Teil- 16 zeitarbeit?

2.2.2 Zunehmende Erwerbsbeteiligung der Frauen. Sind Frauen die eigentlichen "Gewinner" des Beschäftigungssystems?

2.2.3 Unzureichende Qualifikation von Frauen oder Unter-der-Qualifikation-Beschäftigung von Frauen?

2.2.4 Die Diskontinuität weiblicher Erwerbsverläufe 39 - ein wichtiger Pfeiler unseres Beschäftigungssystems

2.2.5 Die geschlechtsspezifische Segregation des Arbeitsmarktes und ihre Hintergrüde

2.2.6 Die Funktion der Frau im Rahmen der geschlechts- 57 spezifischen Arbeitsteilung 
3. Die Bedeutung beruflicher QualifizierungsmaBnahmen für Frauen - keine zentrale, aber eine notwendige Bedingung

4. Anspruch und Möglichkeiten aktiver Arbeitsmarkt- 73 politik in Form beruflicher Qualifizierung nach dem Arbeitsförderungsgesetz

4.1 Leitideen "aktiver" Arbeitsmarktpolitik nach dem AFG und der zeitliche Entstehungszusammenhang des Gesetzes

4.2 Gesamtwirtschaftliche und individuelle Wirkungen "aktiver" Arbeitsmarktpolitik in Form beruflicher Weiterbildung vor dem Hintergrund der derzeitigen Beschäftigungskrise

4.2.1 Reaktiver statt prophylaktischer und prozyklischer statt antizyklischer Einsatz beruflicher Qualifizierungsmaßnahmen

4.2.2 Die gegenwärtige Beschäftigungskrise - eine direkte Folge qualifikatorischer Mängel der Arbeitnehmer?

4.2.3 Technologischer Wandel und Qualifikationsent- 90 wicklung

4.2.4 Hauptfunktion staatlicher Qualifizierungspoli-
tik: GleichmäBigere Verteilung beruflicher
Chancen und Risiken der Arbeitnehmer

4.2.4 Hauptfunktion staatlicher Qualifizierungsp
tik: GleichmäBigere Verteilung beruflicher
Chancen und Risiken der Arbeitnehmer

4.2.5 Weitere Funktionen staatlicher Qualifizierungspolitik
4.2.5.1 Erhalt von Qualifikationen und sozio-kulturel-
len Fähigkeiten
4.2.5.1 Erhalt von Qualifikationen und sozio-kulturel-
len Fähigkeiten

4.2.5.2 Minderung psycho-sozialer Belastungen durch Ar- 98 beitslosigkeit

4.2.5.3 Auslösung erhöhter Weiterbildungsmotivation

4.2.5.4 Korrektiv zum System beruflicher Erstausbildung

4.2 .6

Akzeptanz staatlicher Qualifizierungspolitik durch die Unternehmen

100

4.2.7

Inhaltiche Struktur der QualifizierungsmaBnahmen

100 
5. Fragestellungen einer Wirkungsanalyse arbeits- 103 marktpolitischer MaBnahmen und ihre Behandlung im Rahmen dieser Untersuchung

6. Die untersuchten MaBnahmekategorien der beruf- 115 lichen Weiterbildung

$\begin{array}{lll}6.1 & \text { Fortbildung } & 115\end{array}$

$\begin{array}{lll}6.2 & 122\end{array}$

$\begin{array}{lll}6.3 & \text { Betriebliche Einarbeitung } & 123\end{array}$

$\begin{array}{lll}6.4 & \text { Auftragsmaßnahmen und freie Maßnahmen } & 125\end{array}$

7. Das Leistungsrecht für die berufliche Bildungs- 128 förderung nach dem Arbeitsförderungsgesetz und seine Entwicklung unter besonderer Berücksichtigung seiner frauenspezifischen Wirkungen

$\begin{array}{lll}7.1 & \text { Frauen als Zielgruppe im Arbeitsförderungsge- } 128\end{array}$ setz

$\begin{array}{lll}7.2 & \text { Das Förderrecht und seine Flexibilität }\end{array}$

7.3 Das geltende Förderrecht im Bereich der beruf- 131 lichen Fortbildung und Umschulung

7.3.1 Die wesentlichen Förderregelungen 131

7.3.1.1 Grundlegende Fördervoraussetzungen nach $\$ 36 \quad 133$ (Anspruchsvoraussetzungen)

7.3.1.2 Notwendige berufliche Praxiszeiten nach $\$ 42 \quad 135$ (Förderungsfähiger Personenkreis)

7.3.1.3 Gewährte Leistungen und Beitragsäquivalenz der 140 Leistungen nach $\$ 44$ (Unterhaltsgeld), \$ 45 (Umfang der Förderung) und $\S 46$ (Weitere Leistungsvoraussetzungen, Rückzahlung)

7.3.1.3.1 Leistungen und versicherungsrechtliche Voraus- 140 setzungen im Überblick

7.3.1.3.2 "Großes" Unterhaltsgeld bei notwendigen Voll- 143 zeitmaßnahmen 
7.3.1.3.3 Teil-Unterhaltsgeld bei notwendigen Teilzeit- 147 maßnahmen

7.3.1.3.4 "Kleines" Unterhaltsgeld bei zweckmäßigen Voll-148 zeitmaßnahmen

7.3.1.3.5 Exkurs: 150

Bemessungsentgelt des "großen" und "kleinen"

Unterhaltsgeldes

7.3.1.3.6 Unterhaltsgeld in Höhe des Arbeitslosengeldes/ 151 der Arbeitslosenhilfe

7.3.1.3.7 Ausschließliche Übernahme der Maßnahmekosten 152

7.3.1.3.8 Zusammenfassung der frauenspezifischen Wirkun- 153 gen der Förderkonditionen

$\begin{array}{lll}7.4 & \text { Das geltende Förderrecht im Bereich der be- } 156\end{array}$ trieblichen Einarbeitung

7.5 Zur Entwicklung des Förderrechts seit der Ver- 158 abschiedung des Arbeitsförderungsgesetzes im Jahre 1969

8. Die Beteiligung von Frauen an MaBnahmen der be- 179 ruflichen Weiterbildung

8.1 Berufliche Bildungsmaßnahmen insgesamt 179

$\begin{array}{lll}8.1 .1 & \text { Situation im Jahre } 1985 & 179\end{array}$

$\begin{array}{lll}8.1 .2 & \text { Entwicklung } & 183\end{array}$

8.2 Verteilung der Teilnehmer/innen auf einzelne 197 Maßnahmearten

8.2.1 Verteilung auf Fortbildung, Maßnahmen zur Ver- 197 besserung der Vermittlungsaussichten, Umschulung und betriebliche Einarbeitung

$\begin{array}{lll}\text { 8.2.1.1 Situation im Jahre } 1985 & 197\end{array}$

8.2.1.2 Entwicklung 211

8.2.2 Die Beteiligung von Frauen an den verschiede- 214

$\begin{array}{lll}\text { 8.2.2.1 Situation im Jahre } 1985 & 214\end{array}$ 
8.2.2.2 Entwicklung 220

8.3 Die Weiterbildung von Frauen nach Berufen 223

8.4 Das Teilnahmeniveau zuvor arbeitsloser Frauen 238 und Männer und seine Entwicklung und die "Entlastungseffekte" für den Arbeitsmarkt

8.4.1 Situation im Jahre $1985 \quad 238$

8.4.2 Arbeitsmarktpolitischer Entlastungseffekt 248

8.4.3 Entwicklung 251

8.4.4 Exkurs: Regionalvergleich 252

8.5 Weiterbildung von Frauen nach der Dauer der 257

8.6 Weiterbildung von Frauen nach dem Träger der 270

8.7 Weiterbildung von Frauen nach der AbschluBart 277

8.8 Weiterbildung von Frauen nach personenspezifi- 286 schen Merkmalen

8.8.1 Die Weiterbildungsteilnahme der "neu oder wie- 286 der" ins Berufsleben eintretenden Frauen - eine der Zielgruppen des AFG

8.8.1.1 Die besondere Förderbedürftigkeit der "neu oder 286 wieder" ins Berufsleben eintretenden Frauen

8.8.1.2 Zur Situation im Jahre $1985 \quad 288$

8.8.1.3 Weitere Strukturmerkmale der "neu oder wieder" 297 ins Berufsleben eintretenden Frauen

8.8.1.4 Entwicklung 300

8.8.2 Weiterbildung von Frauen nach dem Familienstand 304

8.8.3 Weiterbildung von Frauen nach dem Alter 306

8.8.4 Weiterbildung von Frauen nach ihrer Qualifi- 316 kation

8.8.4.1 Die besondere Förderbedürftigkeit der minder- $\quad 316$ qualifizierten Frauen 
8.8.4.2 Weiterbildung von Frauen nach ihrer beruflichen 319 Vorbildung

8.8.4.3 Weiterbildung von Frauen nach ihrer schulischen 331 Vorbildung

8.8.4.4 Weiterbildung von Frauen nach der Stellung im 334 Beruf vor Eintritt in die Maßnahme

8.8.4.5 Ursachen für die geringe Beteiligung minder351 qualifizierter Frauen und Männer an beruflichen Weiterbildungsmaßnahmen

9. Die Wirksamkeit der Weiterbildungsförderung von 362 Frauen

9.1 Weiterbildung von Frauen und Schulungserfolg 362

9.2 Berufliche Weiterbildung von Frauen und Berufs- 384 verlauf nach AbschluB der beruflichen Qualifizierungsmaßnahmen

9.2.1 Wiedereingliederungsgrad nach AbschluB der Wei- 384 terbildung

9.2.2 Weiterbildungsadäquanz der Beschäftigung nach 409 AbschluB der Weiterbildung

10. Zur Berücksichtigung von Frauen bei den inner- 414 betrieblichen QualifizierungsmaBnahmen und den Einarbeitungshilfen des Arbeitsmarktpolitischen Sonderprogramms von 1979

11. Frauen in Allgemeinen MaBnahmen zur Arbeits- 426 beschaffung ( $A B M)$

11.1 Die besondere Stellung der ABM im System akti- 426 ver Arbeitsmarktpolitik nach dem $A F G$

11.2 Beschäftigungspolitische und ökonomische Aspek- 428 te von $A B M$ in gesamtwirtschaftlicher Sicht

11.3 Individuelle Auswirkungen der ABM-Teilnahme 434

11.4 Das Förderrecht im Bereich der Allgemeinen MaB-441 nahmen zur Arbeitsbeschaffung unter besonderer Berücksichtigung seiner frauenspezifischen Wirkungen 
11.4.1 Das Förderrecht im Bereich der Standardförderung nach dem AFG

11.4.2 Das Förderrecht der Sonderprogramme, insbesondere des arbeitsmarktpolitischen Programms der Bundesregierung für Regionen mit besonderen Beschäftigungsproblemen

11.5 Die Beteiligung von Frauen an $A B M$

11.5.1 Das Teilnahmeniveau von Frauen im Jahre 1985 und seine Entwicklung zwischen 1978 und 1985

11.5.2 Die Beteiligung von Frauen an ABM nach MaB469

11.5.3 Die Beteiligung der arbeitsmarktpolitischen Zielgruppen an $A B M$

11.5.4 Auswirkungen der ABM-Teilnahme auf die Qualifikation der geförderten Frauen

11.5.5 Die Wirkung der ABM-Teilnahme auf die beruflichen Wiedereingliederungschancen der geförderten Frauen

11.6 Die Berücksichtigung von Frauen im ABM-Förderteil des arbeitsmarktpolitischen Sonderprogramms von 1979

11.7 Ursachen für die Unterrepräsentation von Frauen 496 in $A B M$

12. Frauen in der Regionalpolitik 500

12.1 Regionale Unterschiede in den Erwerbsmöglich- 500 keiten für Frauen

12.2 Die Fördermodalitäten der Gemeinschaftsaufgabe 504 "Verbesserung der regionalen Wirtschaftsstruktur" und ihre frauenspezifischen Wirkungen

12.3 Die Förderung von Frauenarbeitsplätzen mit Mitteln der Gemeinschaftsaufgabe "Verbesserung der regionalen Wirtschaftsstruktur" 
13. Schlubfolgerungen 524

13.1 Zum Handlungsspielraum 524

13.2 Möglichkeiten zur Verstärkung der Beteiligung 527 von Frauen an staatlich geförderten Qualifizierungsmaßnahmen

13.3 Möglichkeiten zur Verstärkung der Beteiligung von Frauen an Arbeitsbeschaffungsmaßnahmen

13.4 Möglichkeiten zur regionalen Förderung der Frauenarbeit 


\title{
VERZEICHNIS DER ÜBERSICHTEN
}

\author{
Übersicht 1 Maßnahmen zur beruflichen Weiterbildung 116 \\ $n$ ach dem AFG \\ Ubersicht 2 Erforderliche berufliche Praxiszeiten für \\ die Förderung der beruflichen Fortbildung \\ und Umschulung nach § 42 AFG \\ Übersicht 3 Höhe und Entwicklung des Unterhaltsgeldes 163 \\ von 1969 bis 1986 \\ Übersicht 4 Programmbedingungen im Schwerpunkt 2 des 420 \\ Sonderprogramms von 1979 \\ Übersicht 5 Ausgewählte Förderkonditionen von ABM seit 443 \\ 1972
}




\section{VERZEICHNIS DER ABBILDUNGEN}

Abbildung 1 Erwerbsquoten verheirateter Frauen nach Alter 1970 und 1984 - Bundesgebiet -

Abbildung 2 Teilnahmequoten der Frauen an beruflichen Bildungsmaßnahmen in V.H. Teilnahmequoten der Männer 1970 bis 1985

Abbildung 3 Eintritte von Frauen und Männern in beruf liche BildungsmaBnahmen 1970 - 1985 und

Teilnehmerbestand jeweils Ende Dezember

eines Jahres - Bundesgebiet -

Abbildung 4 Eintritte von Frauen und Männern in beruf- 190 liche Bildungsmaßnahmen 1970 - 1985 und

Teilnehmerbestand jeweils Ende Dezember

eines Jahres - Nordrhein-Westfalen -

Abbildung 5 Variationsbereiche regionaler Erwerbsquoten 501 nach Altersgruppen 


\section{VERZEICHNIS DER TABELLEN}

Tab. 1 Geschlechtsspezifische Entwicklung der re- 6 gistrierten Arbeitslosigkeit $\begin{array}{lll}\text { Tab. } 2 & \text { Altersspezifische Arbeitslosenquoten von } \\ & \text { Frauen und Männern im Jahre 1985 - Nordrhein- }\end{array}$ Westfalen -

Tab. 3 Stille Reserve nach Alter und Geschlecht $1982 \quad 8$

Tab. 4 Arbeitslose Frauen und Männer nach dem Lei- 11 stungsbezug in V.H. (September 1985) - Bundesgebiet -

Tab. 5 Anteil der Dauerarbeitslosen an den Arbeitslo- 13 sen nach Geschlecht und Alter im September 1985 - Bundesgebiet -

Tab. 6 Teilzeitarbeitslosenquote von Frauen und Voll- 18 zeitarbeitslosenquote von Frauen und Männern

Tab. 7 Erwerbsquoten von Frauen nach dem Alter 21

Tab. 8 Erwerbsquoten verheirateter Frauen nach dem 23 Alter

Tab. 9 Erwerbstätigenquoten von Müttern mit Kindern 25 unter 18 Jahren - Bundesgebiet -

Tab. 10 Erwerbstätigenquoten von Müttern mit Kindern 25 nach dem Alter im Jahre 1982 - Bundesgebiet -

Tab. 11 Frauenanteil an den sozialversicherungspflich- 28 tig beschäftigten Arbeitnehmern

Tab. 12 Qualifikationsspezifische Merkmale von arbeits- 33 losen Frauen und Männern - Bundesgebiet -

Tab. 13 Verteilung von weiblichen und männlichen Arbeitern und Angestellten auf Leistungsgruppen im Januar 1986 - Nordrhein-Westfalen -

Tab. 14 Arbeitslose Frauen und Männer nach ihrem erwerbswirtschaftlichen Status vor der Arbeitslosmeldung im September 1985 - Bundesgebiet - 
Tab. 15 Sozialversicherungspflichtig beschäftigte Frauen und arbeitslose Frauen nach ihrer Verteilung auf Berufsordnungen sowie Frauenanteil an den Beschäftigten und Arbeitslosen 1985 - Bundesgebiet -

Tab. 16 Sozialversicherungspflichtig beschäftigte Frauen am 30.6.1984 nach ihrer Verteilung auf Berufsordnungen - Nordrhein-Westfalen -

Tab. 17 Arbeitslosigkeit von Frauen und Männern die unmittelbar vor Eintritt der Arbeitslosigkeit erfolgreich eine berufliche Ausbildung abgeschlossen haben, in den 10 am häufigsten absolvierten weibiichen Ausbildungsberufen

Tab. 18 Arbeitslosigkeit von Frauen, die erfolgreich eine berufliche Ausbildung in einem "Männerberuf" abgeschlossen haben (1983) - Bundesgebiet -

Tab. 19 Die finanziellen Aufwendungen der Bundesanstalt für Arbeit für Maßnahmen der beruflichen Fortbildung, Umschulung und betrieblichen Einarbeitung $1970-1985$

Tab. 20

In Fortbildungs - und Umschulungsmaßnahmen eingetretene Frauen nach dem Unterhaltsgeldbezug und der Maßnahmeart im Jahre 1983 - NordrheinWestfalen -

Tab. 21 Höhe des Unterhaltsgeldes und Differenz zum Nettoarbeitsentgelt, zum Arbeitslosengeld und zur Arbeitslosenhilfe

Tab. 22 Durchschnittlicher Unterhaltsgeldbetrag von Frauen und Männern nach Alter und Familienstand im Oktober 1985 - Bundesgebiet -

Tab. $23 \mathrm{~N}$ Die in berufliche Bildungsmaßnahmen neu einge- 180 tretenen Männer und Frauen 1970 - 1985 - Nordrhein-Westfalen -

Tab. 23 B Die in berufliche Bildungsmaßnahmen neu einge- 181 tretenen Männer und Frauen 1970 - 1985 - Bundesgebiet -

Tab. 23 NT Teilnehmer an beruflichen Bildungsmaßnahmen 1969 - 1985 - Nordrhein-Westfalen -

Tab. 23 BT Teilnehmer an beruflichen Bildungsmaßnahmen 1969 - 1985 - Bundesgebiet - 
Tab. $24 \mathrm{~N}$ Die in berufliche Bildungsmaßnahmen neu einge- 198 tretenen Frauen nach der Art der Maßnahme 1970 - 1985 - Nordrhein-Westfalen -

Tab. 24 B Die in berufliche Bildungsmaßnahmen neu einge- 199 tretenen Frauen nach der Art der Maßnahme

1970 - 1985 - Bundesgebiet -

Tab. $25 \mathrm{~N}$ Eintritte von Männern und Frauen in berufli- 200 liche Bildungsmaßnahmen nach ihrer prozentualen Verteilung auf einzelne Maßnahmearten

1970 - 1985 - Nordrhein-Westfalen -

Tab. 25 B Eintritte von Männern und Frauen in berufliche 201 Bildungsmaßnahmen nach ihrer prozentualen Verteilung auf einzelne Maßnahmearten 1970 1985 - Bundesgebiet -

Tab. 25 NT Teilnehmer an beruflichen BildungsmaBnahmen und ihre prozentuale Verteilung auf einzelne Maßnahmearten 1971 - 1985 - Nordrhein-Westfalen -

Tab. 25 BT Teilnehmer an beruflichen BildungsmaBnahmen und ihre prozentuale Verteilung auf einzelne Maßnahmearten 1971 - 1985 - Bundesgebiet -

Tab. 26

Differenz zwischen dem Anteil der Frauen an den abhängigen Erwerbspersonen und ihrem Anteil an den Neueintritten in berufliche $B i 1$ dungsmaßnahmen im Jahre 1985 in Prozentpunkten

Tab. 26 T Differenz zwischen dem Anteil der Frauen an den abhängigen Erwerbspersonen und ihrem Anteil an den Teilnehmern (Bestände) im Jahre 1985 in Prozentpunkten

Frauenanteil an den einzelnen beruflichen $B i 1-207$ dungsmaßnahmen, Eintritte von Frauen in \% der Eintritte von Männern und Frauen insgesamt 1970 - 1985 - Nordrhein-Westfalen

Tab. 27 B Frauenanteil an den einzelnen beruflichen Bil- 208 dungsmaßnahmen, Eintritte von. Frauen in $\%$ der Eintritte von Männern und Frauen insgesamt 1970 - 1985 - Bundesgebiet -

Tab. 27 NT Frauenanteil an den einzelnen beruflichen Bil- 209 dungsmaßnahmen, Weibliche Teilnehmer in Prozent aller Teilnehmer 1969 - 1985 - NordrheinWestfalen - 
Tab. 27 BT Frauenanteil an den einzelnen beruflichen Bil- 210 dungsmaßnahmen, Weibliche Teilnehmer in Prozent aller Teilnehmer 1969 - 1985 - Bundesgebiet -

Tab. $28 \mathrm{~N}$ Die in berufliche Fortbildungsmaßnahmen eingetretenen Männer und Frauen nach dem $\mathrm{Ziel}$ der Maßnahme 1978 - 1985 - Nordrhein-Westfalen -

Tab. 28 B Die in berufliche Fortbildungsmaßnahmen eingetretenen Männer und Frauen nach dem Ziel der Maßnahme 1973 - 1985 - Bundesgebiet -

Tab. $29 \mathrm{~N}$ Die acht am stärksten besetzten Schulungsziele (Berufsgruppen) von Frauen im Jahre 1983 - Nordrhein-Westfalen -

Tab. 30 N Die acht am stärksten besetzten Schulungsziele (Berufsgruppen) von Männern im Jahre 1983 - Nordrhein-Westfalen -

Tab. 29 B Die acht am stärksten besetzten Schulungsziele (Berufsgruppen) von Frauen im Jahre 1984 - Bundesgebiet -

Tab. 3o B Die acht am stärksten besetzten Schulungsziele (Berufsgruppen) von Männern im Jahre 1984 - Bundesgebiet -

Tab. $31 \mathrm{~N}$ Die acht am stärksten besetzten Berufsgruppen bei den sozialversicherungspflichtig beschäftigten Frauen zum 30.6.1984 - Nordrhein-Westfalen -

Tab. $32 \mathrm{~N}$ Uberrepräsentierte und unterrepräsentierte Schulungsziele bei Frauen - Nordrhein-Westfalen -

Tab. 33 B Die acht am stärksten besetzten Berufsgruppen bei den arbeitslosen Frauen Ende September 1984 - Bundesgebiet -

Tab. $34 \mathrm{~N}$ Die zehn am stärksten besetzten Umschulungsziele (Berufsordnungen) für Frauen im Jahre 1983 (neu eingetretene Personen) - NordrheinWestfalen -

Tab. $35 \mathrm{~N}$ Die am stärksten besetzten Umschulungsziele (Berufsordnungen) für Männer im Jahre 1983 (neu eingetretene Personen) - Nordrhein-Westfalen - 
Tab. $36 \mathrm{~N}$ Anteil von zuvor Arbeitslosen an den in beruf- 239 liche Bildungsmaßnahmen neu eingetretenen Männern und Frauen und zuvor arbeitslose Männer und Frauen in Prozent aller Arbeitslosen 1970 - 1985 - Nordrhein-Westfalen -

Tab. 36 B Anteil von zuvor Arbeitslosen an den in beruf- 240 liche Bildungsmaßnahmen neu eingetretenen Männern und Frauen und zuvor arbeitslose Männer und Frauen in Prozent aller Arbeitslosen 1970 - 1985 - Bundesgebiet -

Tab. 36 NT Anteil von zuvor Arbeitslosen an den Teilneh241 mern an beruflichen BildungsmaBnahmen und zuvor arbeitslose Teilnehmer in Prozent aller Arbeitslosen 1978 - 1985 - Nordrhein-Westfalen

Tab. 36 BT Anteil von zuvor Arbeitslosen an den Teilnehmern an beruflichen BildungsmaBnahmen und zuvor arbeitslose Teilnehmer in Prozent aller Arbeitslosen 1978 - 1985 - Bundesgebiet -

Tab. 37 Anteil zuvor arbeitsloser Männer und Frauen an 244 den neu eingetretenen Teilnehmern/innen im Jahre 1983 in den Maßnahmearten der beruflichen Fortbildung

Tab. $38 \mathrm{~N}$ Personen, die vor Eintritt in die Maßnahme ar- 247 beitslos waren, nach der Dauer der Arbeitslosigkeit 1980 - 1985 - Nordrhein-Westfalen -

Tab. 38 B Personen, die vor Eintritt in die Maßnahme ar- 247 beitslos waren, nach der Dauer der Arbeitslosigkeit 1980 - 1985 - Bundesgebiet -

Tab. 39 Höhe der Arbeitslosigkeit unter Berücksichtigung zuvor arbeitsloser Teilnehmer (Bestandsdaten) an beruflichen Bildungsmaßnahmen und Beschäftigter in ArbeitsbeschaffungsmaBnahmen ( $A B M)$ im Jahre 1985

Tab. 40 Teilnehmer (Neueintritte im Laufe des Jahres 1984) an beruflichen BildungsmaBnahmen und Arbeitslose 1984 nach ihrer Verteilung auf Landesarbeitsamtsbezirke sowie Arbeitslosenquoten nach Landesarbeitsamtsbezirken und Geschlecht

Tab. $41 \mathrm{~N}$ Arbeitsamtsbezirke in Nordrhein-Westfalen mit
den höchsten und niedrigsten weiblichen Teil-

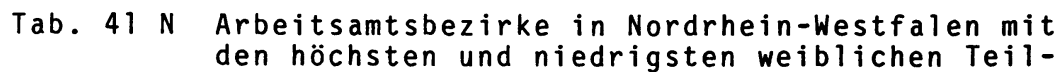
nahmequoten an beruflichen Bildungsmaßnahmen im Jahre 1983 
Tab. $42 \mathrm{~N}$ Die in berufliche BildungsmaBnahmen neu einge- 259 tretenen Frauen und Männer nach der Dauer der Maßnahme 1978 - 1985 - Nordrhein-Westfalen -

Tab. 42 B Die in berufliche Bildungsmaßnahmen neu einge- 261 tretenen Frauen und Männer nach der Dauer der Maßnahme 1971 - 1985 - Bundesgebiet -

Tab. 42 NT Weibliche und männliche Teilnehmer an beruflichen BildungsmaBnahmen nach der Dauer der Maßnahme 1980 - 1985 - Nordrhein-Westfalen -

Tab. 42 BT Weibliche und männliche Teilnehmer an beruflichen Bildungsmaßnahmen nach der Dauer der Maßnahme 1980 - 1985 - Bundesgebiet -

Tab. $43 \mathrm{~N}$ Die in berufliche Bildungsmaßnahmen neu eingetretenen Männer und Frauen nach Dauer und Art der Maßnahme 1978 - 1985 - Nordrhein-Westfalen -

Tab. 43 B Die in berufliche Bildungsmaßnahmen neu eingetretenen Männer und Frauen nach Dauer und Art der Maßnahme 1971 - 1985 - Bundesgebiet -

Die in berufliche Bildungsmaßnahmen eingetre-

tenen Männer und Frauen nach dem Träger der

Maßnahme 1980 - 1985 - Nordrhein-Westfalen -

Tab. 44 B Die in berufliche Bildungsmänahmen eingetretenen Männer und Frauen nach dem Träger der Maßnahme 1980 - 1985 - Bundesgebiet -

Tab. $45 \mathrm{~N}$ Austritte mit Prüfung in v.H. Austritte, bei denen das Schulungsziel als erreicht galt

- Nordrhein-Westfalen.

Tab. 45 B Austritte mit Prüfung in v.H. Austritte, bei denen das Schulungsziel als erreicht galt

- Bundesgebiet -

Tab. $46 \mathrm{~N}$ Männer und Frauen, die eine berufliche Fortbildungs - oder Umschulungsmaßnahme erfolgreich mit einer Prüfung abgeschlossen haben, nach Art der Prüfung 1971 - 1985 - Nordrhein-Westfalen -

Tab. 46 B Männer und Frauen, die eine berufliche Fortbildungs - oder Umschulungsmaßnahme erfolgreich mit einer Prüfung abgeschlossen haben, nach Art der Prüfung 1971-1985 - Bundesgebiet - 
Tab. $47 \mathrm{~N}$ Die in berufliche Bildungsmaßnahmen eingetre290 tenen Männer und Frauen, die neu oder wieder ins Berufsleben eintreten wollten - NordrheinWestfalen -

Tab. 47 B Die in berufliche Bildungsmaßnahmen eingetretenen Männer und Frauen, die neu oder wieder ins Berufsleben eintreten wollten - Bundesgebiet -

Tab. 48 B Zahl der Frauen, die neu oder wieder in das Berufsleben eintreten wollten, und ihr Anteil an allen weiblichen Neueintritten - Bundesgebiet -

Tab. 49 Anteil von in den letzten 6 Jahren nicht erwerbstätigen und von bisher nicht erwerbstätigen Frauen an den Teilnehmerinnen an Maßnahmen zur Verbesserung der Vermittlungsaussichten gem. $\S 41$ a $A F G$

Tab. 50 Ausgewählte Strukturmerkmale von Frauen, die vor Eintritt in berufliche Bildungsmaßnahmen sechs oder mehr Jahre oder bisher noch nicht berufstätig waren

Tab. 51 Anteil alleinstehender Personen an den neu in berufliche Bildungsmaßnahmen eingetretenen Teilnehmern/innen und an den Arbeitslosen

Die in berufliche Bildungsmaßnahmen eingetretenen Männer und Frauen nach Altersgruppen 1980 - 1985 - Nordrhein-Westfalen -

Tab. 52 B Die in berufliche Bildungsmaßnahmen eingetretenen Männer und Frauen nach Altersgruppen 1980 - 1985 - Bundesgebiet -

Tab. $53 \mathrm{~N}$ Zuvor arbeitslose (neu eingetretene) Teilnehmer/innen an beruflichen Bildungsmaßnahmen insgesamt (einschließlich Maßnahmen zur Verbesserung der Vermittlungsaussichten) in v.H. der Arbeitslosen nach Altersgruppen - Nordrhein-Westfalen -

Tab. $54 \mathrm{~N}$ Zuvor arbeitslose (neu eingetretene) Teilnehnehmer/innen an beruflichen Bildungsmaßnahmen insgesamt (einschließlich Maßnahmen zur Verbesserung der Vermittlungsaussichten) in v.H. der Arbeitslosen nach Altersgruppen - Bundesgebiet - 
Tab. 55 Verteilung der zuvor arbeitslosen Teilnehmerinnen an beruflichen BildungsmaBnahmen auf MaBnahmearten und Altersklassen im Jahre 1983

Tab. $56 \mathrm{~N}$ Die in berufliche BildungsmaBnahmen eingetretenen Männer und Frauen nach ihrer Schul- und Berufsausbildung 1980 - 1985 - Nordrhein-Westfalen -

Tab. 56 B Die in berufliche Bildungsmaßnahmen eingetretenen Männer und Frauen nach ihrer Schul- und Berufsausbildung 1980 - 1985 - Bundesgebiet -

Tab. 57 B Zuvor arbeitslose (neu eingetretene) Teilnehmer/innen in beruflichen Bildungsmaßnahmen (insgesamt) sowie Arbeitslose nach der beruflichen Vorbildung - Bundesgebiet -

Tab. $58 \mathrm{~N}$ Anteil der Männer und Frauen ohne abgeschlossene Berufsausbildung an den Arbeitslosen und an den neu in Umschulungs- und betriebliche Einarbeitungsmaßnahmen eingetretenen Teilnehmern - Nordrhein-Westfalen -

Tab. $59 \mathrm{~N}$ Die in berufliche Bildungsmaßnahmen (insgesamt) neu eingetretenen Männer und Frauen nach der Stellung im Beruf vor der Maßnahme 1980 - 1985 - Nordrhein-Westfalen -

Tab. 59 B Die in berufliche Bildungsmaßnahmen (insgesamt) neu eingetretenen Männer und Frauen nach der stellung im Beruf vor der Maßnahme 1971 - 1985 - Bundesgebiet -

Tab. 6o B Anteile von Nichtfacharbeitern/innen an den neu in Weiterbildungsmaßnahmen eingetretenen Teilnehmern/innen und an den Arbeitslosen - Bundesgebiet -

Tab. 61 B Weiterbildungsquoten zuvor arbeitsloser Teilnehmer/innen nach der Stellung im Beruf vor der MaBnahme im Jahre 1984 - Bundesgebiet -

Tab. 62 B Weiterbildungsquote zuvor nicht arbeitsloser Teilnehmer/innen nach der Stellung im Beruf vor der MaBnahme im Jahre 1984 - Bundesgebiet -

Tab. $63 \mathrm{~N}$ Die in Fortbildungsmaßnahmen (ohne Maßnahmen nach $\S 41$ a) eingetretenen Männer und Frauen nach der Stellung im Beruf vor der Maßnahme 1980 - 1985 - Nordrhein-Westfalen - 
Tab. $64 \mathrm{~N}$ Die in Umschulungsmaßnahmen eingetretenen

Männer und Frauen nach der Stellung im Beruf vor der Maßnahme 1980 - 1985 - NordrheinWestfalen -

Tab. $65 \mathrm{~N}$ Die in betriebliche Einarbeitungsmaßnahmen eingetretenen Männer und Frauen nach der Stellung im Beruf vor der Maßnahme 1980 - 1985

- Nordrhein-Westfalen -

Tab. 63 B Die in Fortbildungsmaßnahmen (ohne Maßnahmen nach $\$ 41$ a) eingetretenen Männer und Frauen nach der Stellung im Beruf vor der Maßnahme 1971 - 1985 - Bundesgebiet -

Tab. 64 B Die in Umschulungsmaßnahmen eingetretenen Männer und Frauen nach der Stellung im Beruf vor der Maßnahme 1971 - 1985 - Bundesgebiet -

Tab. 65 B Die in betriebliche Einarbeitungsmaßnahmen eingetretenen Männer und Frauen nach der Stellung im Beruf vor der Maßnahme 1971- 1985 - Bundesgebiet -

Tab. 66 Teilnahmequoten im Bereich Umschulung nach der 350 Stellung im Beruf vor der Maßnahme im Jahre 1985

Tab. 67 Teilnahmequoten im Bereich "betriebliche Einarbeitung" nach der Stellung im Beruf vor der Maßnahme im Jahre 1985

Tab. $68 \mathrm{~N}$ Die aus beruflichen Bildungsmaßnahmen ausgetretenen Männer und Frauen nach dem Ergebnis der Maßnahme 1971 - 1985 - Nordrhein-Westfalen

Tab. 68 B Die aus beruflichen Bildungsmaßnahmen ausgetretenen Männer und Frauen nach dem Ergebnis der Maßnahme 1971 - 1985 - Bundesgebiet -

Tab. $69 \mathrm{~N}$ Die aus beruflichen Bildungsmaßnahmen ausgetretenen Männer und Frauen nach der Art der MaBnahme und dem Ergebnis 1971 - 1985 - Nordrhein-Westfalen -

Tab. 69 B Die aus beruflichen Bildungsmaßnahmen ausgetretenen Männer und Frauen nach der Art der Maßnahme und dem Ergebnis 1971 - 1985 - Bundesgebiet -

Tab. $70 \mathrm{~N}$ Aus beruflichen Fortbildungsmaßnahmen ausgetretene Männer und Frauen, die das Schulungsziel nicht erreicht haben, nach Fortbildungsarten in V.H. aller Austritte im Jahre 1983

- Nordrhein-Westfalen - 
Tab. $71 \mathrm{~N}$ Aus UmschulungsmaBnahmen ausgetretene Männer und Frauen, die das Schulungsziel nicht erreicht haben, nach ihrer schulischen und beruflichen Vorbildung und ihrer Stellung im Beruf vor Antritt der MaBnahme in v.H. der Austritte im Jahre 1983 - Nordrhein-Westfalen -

Tab. $72 \mathrm{~N}$ Aus UmschulungsmaBnahmen ausgetretene Personen, die das Schulungsziel nicht erreicht haben, in V.H. aller Austritte nach dem Alter im Jahre 1983 - Nordrhein-Westfalen -

Tab. $73 \mathrm{~N}$ Aus den 10 am stärksten besetzten Umschulungs- 379 berufen ausgetretene Frauen, die das Schulungsziel nicht erreicht haben, in v.H. der Austritte im Jahre 1983 - Nordrhein-Westfalen -

Tab. $74 \mathrm{~N}$ Männer und Frauen, die vorzeitig aus einer Bildungsmaßnahme ausgetreten sind, nach den Gründen für das vorzeitige Ausscheiden

- Nordrhein-Westfalen -

Tab. 75 B Anteil der (zuvor arbeitslosen) Personen (in v.H.), die am Ende des nächsten Quartals (nach dem Quartal des Maßnahmeabschlusses) eine Arbeit aufgenommen hatten, unter den Teilnehmer/innen, die im Jahre 1984 erfolgreich eine Bildungsmaßnahme beendet hatten - Bundesgebiet -

Tab. $76 \mathrm{~N}$ Anteil der Personen (in v.H.), die bis 30.6 . 1983 eine Beschäftigung aufgenommen hatten, unter den (vorher arbeitslosen) Teilnehmern/ innen an einer Weiterbildungsmaßnahme, die diese Maßnahme im 1. Quartal 1983 beendet hatten - Nordrhein-Westfalen -

Tab. 76 B Anteil der Personen (in V.H.), die bis 30.6 . 1983 eine Beschäftigung aufgenommen hatten, unter den (vorher arbeitslosen) Teilnehmern/ innen an einer Weiterbildungsmaßnahme, die diese Maßnahme im 1. Quartal 1983 beendet hatten - Bundesgebiet -

Tab. $77 \mathrm{~N}$ Frauen und Männer, die bis zum 30.6.1983 eine Arbeit aufgenommen hatten, in v.H. aller Frauen und Männer, die im 1. Quartal 1983 erfolgreich eine Anpassungs-FortbildungsmaBnahme beendet hatten, nach Schulungszielen - geordnet nach der beruflichen Wiedereingliederungsquote - Nordrhein-Westfalen - 
Tab. 78 Anteil der Personen (in v.H.), die bis zum

3o.6.1983 eine Arbeit aufgenommen hatten, unter den (zuvor arbeitslosen) Teilnehmern/innen, die im 1. Quartal 1983 erfolgreich eine Anpassungs-Fortbildungsmaßnahme beendet hatten, nach Schulungszielen

Tab. $79 \mathrm{~N}$ Frauen und Männer, die bis zum 30.6.1983 eine Arbeit aufgenommen hatten, in v.H. aller (zuvor arbeitslosen) Umschüler/innen, die im 1. Quartal 1983 erfolgreich eine Umschulungsmaßnahme beendet hatten, nach Schulungszielen (geordnet nach der beruflichen Wiedereingliederungsquote) - Nordrhein-Westfalen -

Tab. 80 Anteil der Personen ( in v.H.), die bis zum 30.6.1983 eine Arbeit aufgenommen hatten, unter den (zuvor arbeitslosen) Personen, die im 1. Quartal 1983 erfolgreich eine Umschulungsmaßnahme beendet hatten, nach Schulungszielen

Tab. 81 Dauer der Arbeitslosigkeit bis zum Erhebungszeitpunkt bei den Arbeitslosen Ende September 1983 in Abhängigkeit von der Teilnahme an beruflicher weiterbildung

Tab. 82 Kennziffern zur Repräsentation von Frauen im Schwerpunkt 1: "Innerbetriebliche Qualifizierungsmaßnahmen" des Sonderprogramms von 1979

Tab. 83 Kennziffern zur Beteiligung von Frauen im Schwerpunkt 2: "Wiedereingliederung von ungelernten und langfristig Arbeitslosen" des Sonderprogramms von 1979

Tab. $84 \mathrm{~N}$ Beschäftigte geförderte Männer und Frauen in allgemeinen MaBnahmen zur Arbeitsbeschaffung 1978 - 1985 - Nordrhein-Westfalen -

Tab. 84 B Beschäftigte geförderte Männer und Frauen in allgemeinen Maßnahmen zur Arbeitsbeschaffung 1978 - 1985 - Bundesgebiet -

Tab. $85 \mathrm{~N}$ Arbeitsamtsbezirke in Nordrhein-Westfalen mit den höchsten und niedrigsten Arbeitslosenquoten von Frauen nach ihren ABM-Aktivitäten

- Nordrhein-Westfalen -

Tab. 86

ABM-induzierte Arbeitsmarktentlastungseffekte: 461 weibliche "Entlastungsquote" in v.H. der männlichen "Entlastungsquote" 
Tab. $87 \mathrm{~N}$ Beteiligung von Frauen an den ABM-Aktivitäten

in den Arbeitsamtsbezirken Nordrhein-Westfalens mit den höchsten und niedrigsten FrauenArbeitslosenquoten 1983

Tab. 88 Ausgaben für Arbeitsbeschaffungsmaßnahmen 1982-1985

Tab. $89 \mathrm{~N}$ Im Laufe des Jahres durchgeführte Arbeitsvermittlungen von Männern und Frauen in allgemeine Maßnahmen zur Arbeitsbeschaffung 1978 1985 - Nordrhein-Westfalen -

Tab. 89 B Im Laufe des Jahres durchgeführte Arbeitsvermittlungen von Männern und Frauen in allgemeine Maßnahmen zur Arbeitsbeschaffung 1978 1985 - Bundesgebiet -

Tab. 90 Verhältnis zwischen im Laufe eines Jahres ver- 468 mittelten und den im Jahresdurchschnitt in $A B M$ beschäftigten Personen

Tab. $91 \mathrm{~N}$ Im Laufe des Jahres vermittelte Männer und Frauen in Arbeitsbeschaffungsmaßnahmen nach ihrer prozentualen Verteilung auf einzelne Maßnahmearten 1982 - 1985 - Nordrhein-Westfalen -

Tab. 91 B Im Laufe des Jahres vermittelte Männer und Frauen in Arbeitsbeschaffungsmaßnahmen nach ihrer prozentualen Verteilung auf einzelne Maßnahmearten 1979 - 1985 - Bundesgebiet -

Tab. 92 B Beschäftigte, geförderte Männer und Frauen in
Arbeitsbeschaffungsmaßnahmen nach ihrer prozentualen Verteilung auf einzelne Maßnahmearten 1979 - 1985 - Bundesgebiet -

Tab. 93 Berücksichtigung arbeitsmarktpolitischer Ziel- 476 gruppen in ABM Ende 4. Quartal 1984

Tab. 94 Berücksichtigung arbeitsmarktpolitischer Ziel- 477 gruppen in ABM Ende 3. Quartal 1986

Tab. 95 Regionen mit der höchsten und niedrigsten Er- 502 werbsbeteiligung der Frauen 1985 (Prognosedaten)

Tab. 96 Geförderte Arbeitsplätze im Zeitraum 1.1.1972 512 - 30.9.1984 
Tab. 97 Geförderte Frauenarbeitsplätze in v.H. aller geförderten Arbeitsplätze im Zeitraum 1.1.1972 $-30.9 .1984$

Tab. 98 Entwicklung des Anteils der geförderten Frauenarbeitsplätze (sämtliche Fälle) an den geförderten Arbeitsplätzen vom 1.1.1972 bis zum 30.9 .1984

Tab. 99 B Entwicklung der Zahl der geförderten Frauenar- 514 beitsplätze im Zeitraum 1.1.1972 bis 31.12 . 1985 - Bundesgebiet -

Tab. 100 B Verteilung der geförderten Frauenarbeitsplätze 515 (alle Fälle) auf Wirtschaftszweige im Zeitraum 1.1.1972 - 30.9.1984 - Bundesgebiet -

Tab. 101 Regionale Aktionsräume mit dem niedrigsten und 518 höchsten Frauenanteil an den geförderten Arbeitsplätzen im Zeitraum 1.1.1972 - 30.9.1984

Tab. 102 Verteilung der im Zeitraum 1.1.1972 bis 30.9. 519 1984 geförderten Frauenarbeitsplätze auf Regionale Aktionsräume

Tab. $103 \mathrm{~N}$ Frauenanteil an den Förderfällen im Zeitraum 1.1 .1972 bis 30.6.1984 und Frauenanteil an den sozialversicherungspflichtig Beschäftigten zum 30.6.1985 in den geförderten Kreisfreien Städten und Landkreisen Nordrhein-Westfalens

Tab. $104 \mathrm{~N}$ Verteilung der geförderten Frauenarbeitsplätze 521 im Zeitraum 1.1.1972 - 30.6.1984 auf Kreisfreie Städte und Landkreise in Nordrhein-Westfalen 